antibodies (anti-CarP). Recently, another AMPA: anti-acetylated protein antibodies (AAPA) have been found in RA patients [1]. The prevalence of AAPA antibodies and their isotypes have yet to be determined. Since isotype profiles reflect the breadth of an immune response, the prevalence of AAPA isotypes in arthritis patients with and without RA can help to understand the relevance of this autoantibody response in RA.

Objectives: To describe the prevalence of AAPA isotypes in arthritis patients with and without RA.

Methods: In 650 RA patients fulfilling the 1987 RA criteria and 555 non-RA arthritis patients from the Leiden Early Arthritis Cohort, baseline serum samples were screened by ELISA for IgG, IgM and IgA to an acetylated- and control peptide that was based upon the CCP-2 backbone. The cutoff for positivity was based on 80 controls (mean $+2 \mathrm{SD}$ ). A sample was considered positive if it was above the cutoff and was 0.1 optical density higher on the acetylated peptide than on the control peptide.

Results: AAPA IgG was found in $36 \%$ of RA patients versus $6.7 \%$ of non-RA arthritis patients (figure 1a). Within RA patients, AAPA IgG antibodies were mostly present in the ACPA-(CCP-2) positive group (64\% in ACPA-positive, compared to $5 \%$ in ACPA-negative). Levels of AAPA IgG and IgA were higher in RA patients than in healthy controls and non-RA arthritis patients (figure 1b), however, surprisingly, no difference in levels was found for IgM.

When isotype profiles in AAPA- positive arthritis patients were compared, patients

Table 1. Anti-acetylated protein antibody (AAPA) isotype overlap in AAPA positive patients.

\begin{tabular}{|c|c|c|}
\hline AAPA isotype & RA patients $(=310) n(\%)$ & Non-RA arthritis patients $(n=106) n(\%)$ \\
\hline $\lg G+\lg M-\lg A-$ & 115 (37.1) & $28(5.1)$ \\
\hline $\lg G-\lg M+\lg A-$ & $52(16.8)$ & $48(8.7)$ \\
\hline IgG-IgM-IgA+ & $14(4.5)$ & $13(2.3)$ \\
\hline $\lg G+\lg M+\lg A-$ & $24(7.7)$ & $3(0.5)$ \\
\hline $\lg G+\lg M-\lg A+$ & 37 (11.9) & $4(0.7)$ \\
\hline $\lg G-\lg M+\lg A+$ & $9(2.9)$ & $8(1.4)$ \\
\hline $\lg G+\lg M+\lg A+$ & $59(19.0)$ & $2(0.4)$ \\
\hline
\end{tabular}

AAPA: anti-acetylated protein antibodies, RA: rheumatoid arthritis

with RA were more often positive for two or more isotypes then patients without RA, and thus displayed considerably more overlap in AAPA isotypes compared to non-RA patients (table 1). Intriguingly, IgM AAPA was the most prevalent isotype in non-RA patients, versus IgG in RA patients.

Conclusion: AAPA are detected in one third of RA patients, and mainly in the ACPA-positive subgroup. The predominance of IgM AAPA in non-RA arthritis patients and healthy controls suggests that healthy persons can develop AAPA IgM without the development of RA. These results also suggest that in healthy individuals, AAPA responses can occur, but do not mature past the IgM-stage, while in RA patients, the AAPA-response does mature and might form a "starting point" for development of other AMPA leading to the concurrent present of several AMPA in disease.

REFERENCES:

[1] Juarez, M., et al., Identification of novel antiacetylated vimentin antibodies in patients with early inflammatory arthritis. Ann Rheum Dis, 2016. 75(6): p. 1099-107.

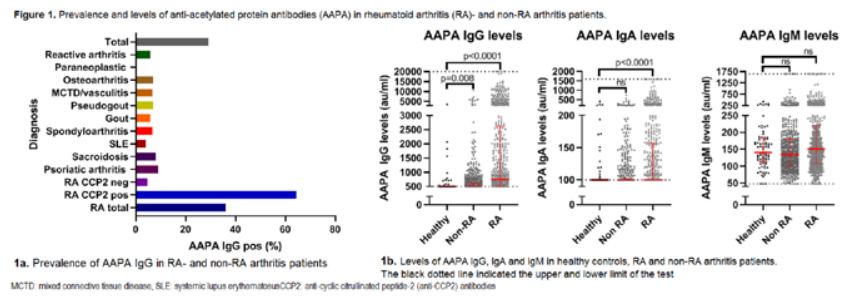

Disclosure of Interests: None declared

DOI: 10.1136/annrheumdis-2021-eular.2777

\section{POS0396 IS ASSOCIATED WITH THE CHANGES OF INTESTINAL MICROBIOTA IN PATIENTS WITH RHEUMATOID ARTHRITIS}

R. Wu ${ }^{1}$, J. An ${ }^{1}$, T. Ding ${ }^{1}$, H. Xue ${ }^{1}$, X. F. Li ${ }^{1}$, C. Wang ${ }^{1} .{ }^{1}$ The Second Hospital of Shanxi Medical University, The Department of Rheumatology, Taiyuan, China

Background: Rheumatoid arthritis (RA) is a systemic autoimmunity inflammation disease characterized with chronic aggressive arthritis and the presence of abnormal antibodies. Several observations showed that the breakdown of immune tolerance caused by many complex interactions was involved in the development of $\mathrm{RA}^{[1]}$. However, the pathogenesis of RA remained unclear. It has been confirmed that the imbalance of Th17 and Treg cells play a crucial role in destroying immune tolerance ${ }^{[2]}$. Besides, researches showed that intestinal microbiota can influence host immunity by acting on the immune cells to play pro-inflammatory or anti-inflammatory effect, and in turn immune system can also regulate the microbiota ${ }^{[3,4]}$. Thus, a frontier point of view in the field of rheumatism, immune microecology, was proposed, which is a novel concept for the breakdown of immune tolerance. Studies have confirmed that there was an imbalance of intestinal microbiota in patients with RA ${ }^{[4]}$. But the relationship between the $C D 4+T$ subsets cells and intestinal microbiota in RA is unknown.

Objectives: We detected and compared the absolute number of CD4+T cells subsets in the peripheral blood and the proportion or abundance of intestina microbiota in patients with RA and healthy adults, and then analyzed the relationship between them to explore the role of CD4+T cells subsets and intestinal microbiota in the pathogenesis of RA.

Methods: We collected the sample of stool and blood from 15 patients with RA hospitalized at the Second Hospital of Shanxi Medical University and 8 age and gender-matched healthy controls $(\mathrm{HC})$. The absolute number of $\mathrm{CD} 4+\mathrm{T}$ cells subsets including Th1, Th2, Th17 and Treg cells were detected by flow cytometry. The 16S rRNA in the stool specimens were sequenced by the Roche/45 high-throughput sequencing platform. We analyzed whether there was correlarion between CD4+T subsets cells and intestinal microbiota.

Results: Patients with RA had a higher level of Christensenellaceae and a lower level of Pseudomonadaceae as compared with those of $\mathrm{HCs}$ at the family level $(p<0.05)$. And at the genus level, the patients with RA had higher levels of Ruminococcus torques, Christensenellaceae R-7, Ruminiclostridium 9 and Ruminococcus 1 compared with those of $\mathrm{HCs}$ $(p<0.05)$ (Figure 1).And the Ruminococcus torques at the genus level was negative correlated with the absolute number of Treg cells $(p<0.001)$ (Figure 2).

Conclusion: The results here suggested that there were different proportion or abundance of intestinal microbiota between the patients with RA andHCs. And the changes of intestinal microbiota such as Ruminococcus torques were associated with Treg cells, further indicating that the imbalance of intestinal microbiota in RA can destory the immune tolerance. The above results uncovered that the intestinal microbiota had immunomodulatory function, which may be the upstream mechanism participated in the pathogenesis of RA.

\section{REFERENCES:}

[1] Weyand CM, Goronzy JJ. The immunology of rheumatoid arthritis. Nat Immunol 2021, 22(1): 10-18.

[2] Weyand CM, Goronzy JJ. Immunometabolism in the development of rheu matoid arthritis. Immunol Rev 2020, 294(1): 177-187.

[3] Brown EM, Kenny DJ, Xavier RJ. Gut Microbiota Regulation of T Cells During Inflammation and Autoimmunity. Annu Rev Immunol 2019, 37: 599-624.

[4] du Teil Espina M, Gabarrini G, Harmsen HJM, Westra J, van Winkelhoff AJ, van Dijl JM. Talk to your gut: the oral-gut microbiome axis and its immunomodulatory role in the etiology of rheumatoid arthritis. FEMS Microbiol Rev 2019, 43(1).
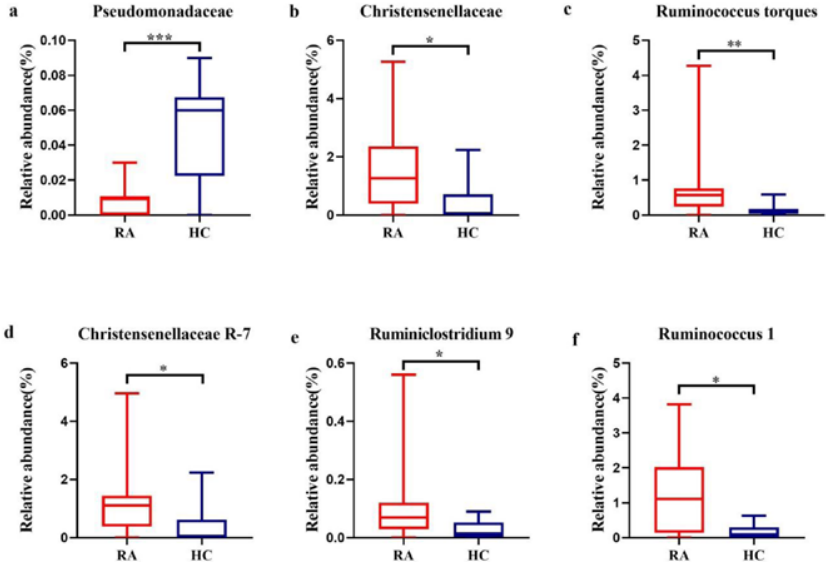

Figure 1. At the family level (a-b) and the genus level(c-f), the relative abundance of intestina microbiota in patients with RA and HCs were different. Data were expressed as median (Q1, Q3) and analyzed by Wilcoxon test. ( ${ }^{\star * *} P<0.001,{ }^{* *} P<0.01$ and $\left.{ }^{*} P<0.05\right)$. 


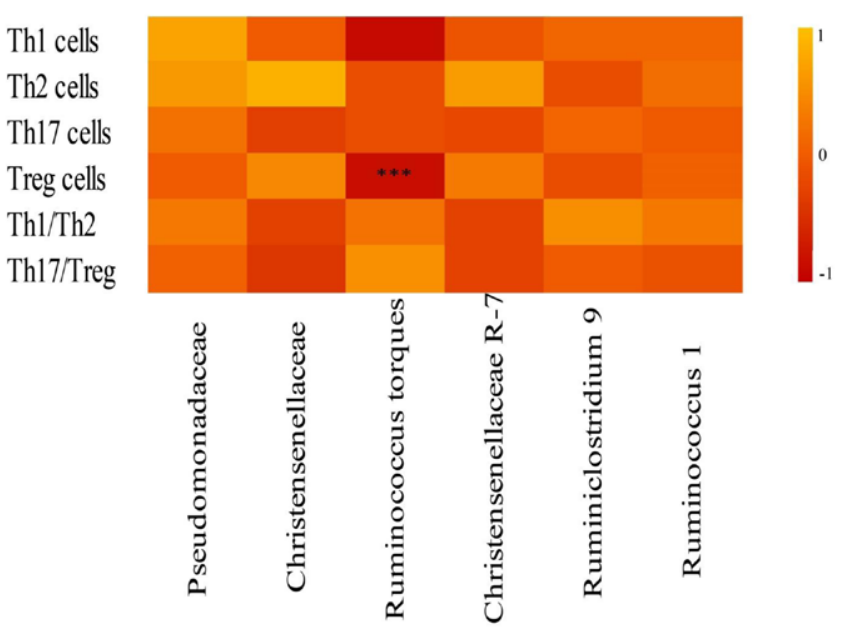

Figure 2. A heatmap shows the correlation between the intestinal microbiota and CD4+T cells in patients with RA, and Ruminococcus torques at the genus level was negative related with Treg cells. (Colors indicate the Spearman rank correlation, ${ }^{\star \star \star} \mathrm{P}<0.001$ ).

Disclosure of Interests: None declared

DOI: 10.1136/annrheumdis-2021-eular.2783

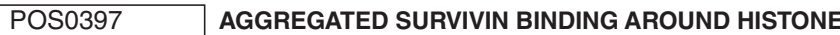 H3 EPIGENETIC MODIFICATIONS IN RISK LOCI ASSOCIATED WITH RHEUMATOID ARTHRITIS}

V. Chandrasekaran ${ }^{1}$, G. Katona ${ }^{2}$, M. I. Bokarewa ${ }^{1}$, K. M. Andersson ${ }^{1}$, M. C. Erlandsson ${ }^{1}$, M. Jensen ${ }^{2}$, N. Oparina ${ }^{1}$, A. Damdimopoulos ${ }^{3}$. ${ }^{1}$ Gothenburg University, Department of Rheumatology, Gothenburg, Sweden;

${ }^{2}$ Gothenburg University, Department of Chemistry and Molecular Biology, Gothenburg, Sweden; ${ }^{3}$ Karolinska Institute, Department of Bioscience and Nutrition, Huddinge, Sweden

Background: Survivin is an integral part of the Chromosomal Passenger Complex (CPC) which plays a vital role in mitosis. Experiments have demonstrated that survivin can physically bind to DNA. Crystallographic studies show that survivin binds to Threonine- 3 of histone $\mathrm{H} 3$. In patients with autoimmune diseases, increased survivin expression contributes to an aggravated disease phenotype. Thus, functional, and mechanistic data point to a potential chromatin regulatory role for survivin, possibly in combination with the established gene regulatory function carried out by histone epigenetic modifications (EM)

Objectives: The objective of the study was to analyse the co-localization of chromatin bound survivin with three histone $\mathrm{H} 3$ epigenetic modifications - acetylated lysine 27 (K27ac) and trimethylated lysine 4 (K4me3) and lysine-27 (K27me3). The second objective was to analyse if survivin-bound DNA sequences overlapped with sequences in the vicinity of 106 GWAS SNPs that are associated with a risk of developing rheumatoid arthritis (RA).

Methods: Chromatin from CD4 T cells of 14 female subjects was immunoprecipitated with survivin antibodies and each of the histone $\mathrm{H} 3$ antibodies, and coupled with sequencing (ChIPseq, Hiseq2000, Illumina). After mapping the annotations of sequenced regions to the human reference genome hg38, enriched peaks were identified through Homer software. The identified survivin ChIP peaks were analysed for colocalization with peaks of the three histone $\mathrm{H} 3 \mathrm{EMs}$ and with RA risk loci, using the Bioconductor package 'ChIPPeakAnno' through RStudio.

Results: Among the total of $\sim 13,000$ individual survivin ChIP-peaks, 33\% colocalized with histone $\mathrm{H} 3 \mathrm{EM}$ peaks. The overlapping peaks show a linear increase in average peak size compared with the peaks showing no colocalization with any $\mathrm{H} 3$ EM peak. A maximum of 5.5 -fold increase in average peak size was observed when survivin bound peaks overlap with peaks of all three H3 EMs. A major proportion (86\%) of top RA risk SNPs was associated with either binding of survivin or $\mathrm{H} 3 \mathrm{EMs}$. In this subset, $63 \%$ of RA risk SNPs were found within an area of 100 kilobases from survivin ChIP-peaks, with preferential enrichment of high-scoring peaks when survivin colocalizes with all $3 \mathrm{H} 3 \mathrm{EMs}$. Survivin was bound to risk SNPs annotated to, among others, the major immunological genes CD83, IRF4, CD28, ICOS and IL2RA
Conclusion: This study presents experimental evidence that survivin binding to DNA preferentially occurred in regions with high density of histone EMs. The increased aggregation of survivin around histone $\mathrm{H} 3 \mathrm{EMs}$ point to its potential regulatory function in gene transcription. Since regions around RA risk SNPs overlap with survivin peaks, survivin's nuclear function could have immunologically important effects in mechanisms of autoimmune diseases.

Disclosure of Interests: None declared

DOI: 10.1136/annrheumdis-2021-eular.3212

\section{POS0398 \\ ADIPONECTIN INDUCES SYNOVIAL ANGIOGENESIS IN RHEUMATOID ARTHRITIS THROUGH METABOLIC REMODELING}

L. Wang ${ }^{1}$, W. Tan ${ }^{1}$, F. Wang ${ }^{2}$, M. Zhang ${ }^{1}{ }^{1}$ The First Affiliated Hospital of Nanjing Medical University, Department of Rheumatology, Nanjing, China; ${ }^{2}$ The First Affiliated Hospital of Nanjing Medical University, Department of Cardiology, Nanjing, China

Background: Our team have previously reported that Adiponectin correlates well with synovial inflammation and progressive bone erosion in rheumatoid arthritis (RA). Angiogenesis is another important part, which plays a critical role in the pathogenesis of RA.

Objectives: We hypothesized that adiponectin induces synovial angiogenesis in RA.

Methods: Single-cell RNA sequencing (scRNA-Seq) was used to screen cellular changes in local knee joint of collagen-induced arthritis (CIA) after intraarticularly injected of adiponectin. Chimera models of synovium-cartilage-NOD/SCID mice, matrigel plug assay and rat aortic ring assay were performed to demonstrate the pro-angiogenesis role of adiponectin. Cellular experiment, including proliferation, migration, apoptosis, tube formation and angiogenesis related gene expression profile, were detected with Human Umbilical Vein Endothelial Cells (HUVEC) and Mice Lung Microvessel Endothelial Cell (MLMEC) after adiponectin stimulation. Seahorse was performed to clear the influence of adiponectin to cell metabolism.

Results: The synovium and pannus hyperplasia worse in CIA model after intraarticularly injected of adiponectin, along with more serious synovitis and bone erosion. ScRNA-Seq of synovial tissues separated from CIA reminded that endothelial cell barbarically grows via metabolic remodeling after stimulated with adiponectin. Synovial chimera, matrigel plug and rat aortic ring shows adiponectin accelerates angiogenesis significantly in different background conditions. In vitro, endothelial cell proliferation detecting by RCTA and CCK8, migration by wound healing and transwell, apoptosis by FACS, tube formation and angiogenesis related gene expression profile by PCR-ARRAY were promoted by adiponectin in both HUVEC and MLMEC Seahorse showed HUVEC made more use of glycolysis after co-cultured with adiponectin, a method of cell energy supply that tumor cells possess called warburg effect, that drives endothelial cell hyperplasia in severe environment.

Conclusion: As a classic metabolic regulator, adiponectin exacerbates CIA by promoting angiogenesis through metabolic remodeling. The findings not only provide a novel insight into the pathogenic role of adiponectin, but also reveals a potential therapeutical strategy to attenuate revascularization in RA.

Disclosure of Interests: None declared

DOI: 10.1136/annrheumdis-2021-eular.3585

\section{\begin{tabular}{|l|l|l|l}
\hline POS0399 TMT-BASED QUANTITATIVE PROTEOMICS & \\
\hline
\end{tabular} ANALYSIS OF SYNOVIAL FLUID-DERIVED EXOSOMES IN RHEUMATOID ARTHRITIS, AXIAL SPONDYLOARTHRITIS, GOUT AND OSTEOARTHRITIS}

Y. Liu ${ }^{1,2}$, Y. Huang ${ }^{1}$, Q. Huang ${ }^{1}$, Z. Huang ${ }^{1}$, Z. Li ${ }^{1}$, T. W. Li ${ }^{1} .{ }^{1}$ Guangdong Second Provincial General Hospital, Department of Rheumatology and Immunology, Guangzhou, China; ${ }^{2}$ University of South China, Guangdong Second Provincial General Hospital, Guangzhou, China

Background: The pathogeneses of the joint diseases rheumatoid arthritis (RA), axial spondyloarthritis (axSpA), gout, and osteoarthritis (OA) are still not fully elucidated. Exosomes in synovial fluid (SF) has a critical role in the pathogenesis of arthritis. None of study has compared the proteomics of SF-derived exosomes in RA, axSpA, gout and OA. 